\title{
A New Synthetic Route to the Formation of Fullerene Superconductors ${ }^{*}$
}

\author{
M. K. Wu and H. Y. Tang
}

Materials Science Center, National Tsing Hua University, Hsinchu, Taiwan, ROC

\section{J. H. Hwang and C. Y. Mou}

Department of Chemistry, National Taiwan University, Taipei, Taiwan ROC

We report a new route to the formation of less air sensitive fullerene superconductors using the mixture of $\mathrm{C}_{60}$ and Alkaline-metal carbonates. The materials so prepared contain oxygen in addition to the alkaline metal dopants. The alkaline metal content determined from single crystal $x$-ray is found to be 2.4 instead of the stoichiometry 3 .

\section{Introduction}

The discovery of fullerene superconductors [1] has attractive great attention for the last three years. However, the highly reactive of the materials with atmosphere hindered the progress for better understanding of this molecular superconducting system. On the other hand, there are propsoals suggesting that the superconducting temperature $\left(T_{c}\right)$ of this molecular system, as that of the high $T_{c}$ cuprate superconductors, may exhibit a maximum if one can vary the carrier concentration [2]. In an attempt to test this suggestion, we discovered a new novel synthetic route to the formation of a less air sensitive fullerene superconductors by mixing oxygen-contain alkaline compounds with $\mathrm{C}_{60}[3]$.

\section{Samples Preparation}

Bulk samples of alkaline carbonates (e.g. $\mathrm{K}_{2} \mathrm{CO}_{3}$ ) doped $\mathrm{C}_{60}$ were prepared by directly mixing appropriate amount of

*support by ROC National Science Council
$\mathrm{C}_{60}$ and alkaline-metal carbonates. The powder or pressed pellets were sealed in a fused quarts. The reactant-containing tube was preheated at $\sim 150^{\circ} \mathrm{C}$ under vacuum to remove moisture before sealing. The tube was heated in a furnace at $200^{\circ} \mathrm{C}$ for one hour, then at $700^{\circ} \mathrm{C}$ for 12 hours.

\section{Results and Discussions}

SEM micrograph and the composition spectra using EDX analyzer of the as grown crystals indicate that the sample contains, in addition to the potassium, also high concentration of oxygen. Single crystal X-ray analyses [3] show that the reacted product of $\mathrm{K}_{2} \mathrm{CO}_{3}+\mathrm{C}_{60}$ has a fcc structure with $14.242(4) \AA$ lattice constant which is almost identical to that of the $\mathrm{K}_{3} \mathrm{C}_{60}$ (14.24(i) [4]). Detailed refinement of the single crystal $\mathrm{x}$-ray data show's that the actual chemical composition found in this work is $\mathrm{K}_{2}{ }_{4} \mathrm{C}_{60}$, instead of the stoichiometry $\mathrm{K}_{3} \mathrm{C}_{60}$ determined by Stephens et al. [4]. 


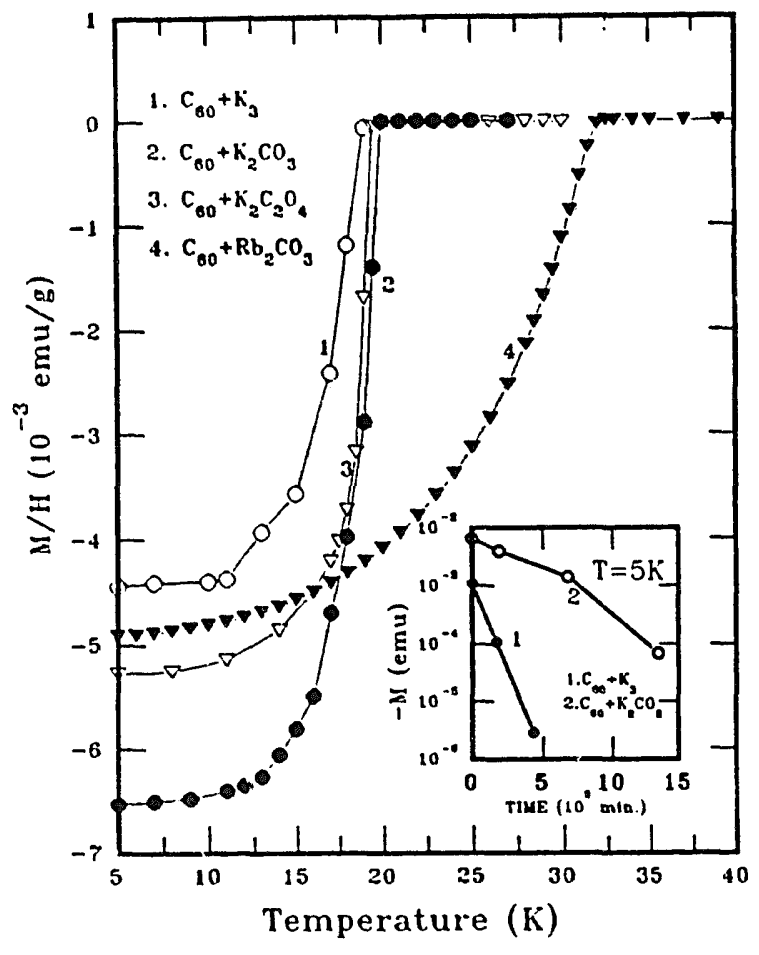

Figure 1. Temperature dependence of the magnetic susceptibility of $\mathrm{K}_{3} \mathrm{C}_{60}$ (1), $\mathrm{C}_{60}+\mathrm{K}_{2} \mathrm{CO}_{3}$ (2), $\mathrm{C}_{60}+\mathrm{K}_{2} \mathrm{C}_{2} \mathrm{O}_{4}$ (3) and $\mathrm{C}_{60}+\mathrm{Rb}_{2} \mathrm{CO}_{3}$ (4); inset are the time dependence of the ZFC signals of samples 1 and 2 .

Figure 1. shows the temperature dependence of the magnetic susceptibility of several $\mathrm{A}_{2} \mathrm{CO}_{3}-\mathrm{C}_{60}$ samples. All measurements were carried out with the sample exposed to air. We have also displayed in the inset of figure 1 the time evolution of the superconducting signals at $5 \mathrm{~K}$, which are measured in zero field cooling, of a sample prepared by mixing $\mathrm{C}_{60}$ with $\mathrm{K}_{2} \mathrm{CO}_{3}$ and the other with $\mathrm{K}$-metal. The $\mathrm{T}_{\mathrm{C}}$ values, determined from the onset of superconducting transition, are found in general about two degrees higher than their corresponding relatives prepared from
Alkaline metals. The other novel result is the air stability of the materials prepared with the carbonates, as shown in the inset of figure 1 . We found the stability of the materials prepared with this new process greatly improved. In fact, we believe the superconducting phase in this sample would actually be stabilized if the samples were not contaminated with the moisture.

The similarity in superconducting transition of the samples prepared by using alkaline-carbonates is surprising since $\mathrm{A}_{2} \mathrm{CO}_{3}$ is not an electron donor. One plausible mechanism is that the Alkalinecarbonate decomposes under the reacting conditions and the released $\mathrm{K}_{2} \mathrm{O}$ complex reacted with the $\mathrm{C}_{60}$ molecules. The less than 3 value of the potassium content may originate from the presence of oxygen which serves as an oxidative agent. The enhancement in the stability of the superconductivity can also be understood in terms of the inclusion of oxygen in these compounds. There were proposals [5] to generate hole doping under oxidative condition. Whether the success in incorporation oxygen to the Alkaline-metal doped superconductors leads to the realization of hole-doping in the fullerenes remains to be corroborated.

\section{References}

1. A. F. Hebard, et al., Nature, 350 (1991) 600.

2. R. Friedberg, et al., Phys. Rev. B46 (1992) 14150.

3. J. H. Hwang, et al., unpublished.

4. P. W. Stephens., et al., Nature, 351 (1991) 632.

5. Q. Zhu, et. al., Nature, 355 (1992) 712 . . 